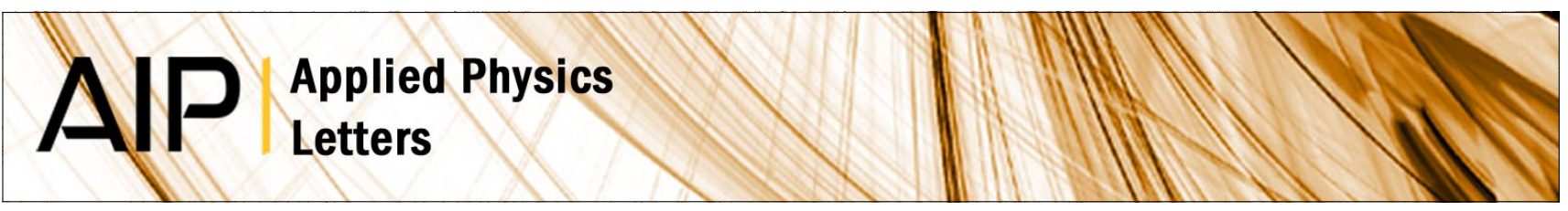

\title{
Origin of thickness dependent dc electrical breakdown in dielectrics
}

George Chen, Junwei Zhao, Shengtao Li, and Lisheng Zhong

Citation: Appl. Phys. Lett. 100, 222904 (2012); doi: 10.1063/1.4721809

View online: http://dx.doi.org/10.1063/1.4721809

View Table of Contents: http://apl.aip.org/resource/1/APPLAB/v100/i22

Published by the American Institute of Physics.

\section{Related Articles}

Impulse breakdown delay in liquid dielectrics

Appl. Phys. Lett. 100, 192910 (2012)

Chemisorption on semiconductors: The role of quantum corrections on the space charge regions in multiple dimensions

Appl. Phys. Lett. 100, 183106 (2012)

Thermal poling of alkaline earth boroaluminosilicate glasses with intrinsically high dielectric breakdown strength J. Appl. Phys. 111, 083519 (2012)

Space charge effect in ultrathin ferroelectric films

J. Appl. Phys. 111, 084103 (2012)

Opto-electric particle manipulation on a bismuth silicon oxide crystal

Appl. Phys. Lett. 100, 161903 (2012)

\section{Additional information on Appl. Phys. Lett.}

Journal Homepage: http://apl.aip.org/

Journal Information: http://apl.aip.org/about/about_the_journal

Top downloads: http://apl.aip.org/features/most_downloaded

Information for Authors: http://apl.aip.org/authors

\section{ADVERTISEMENT}

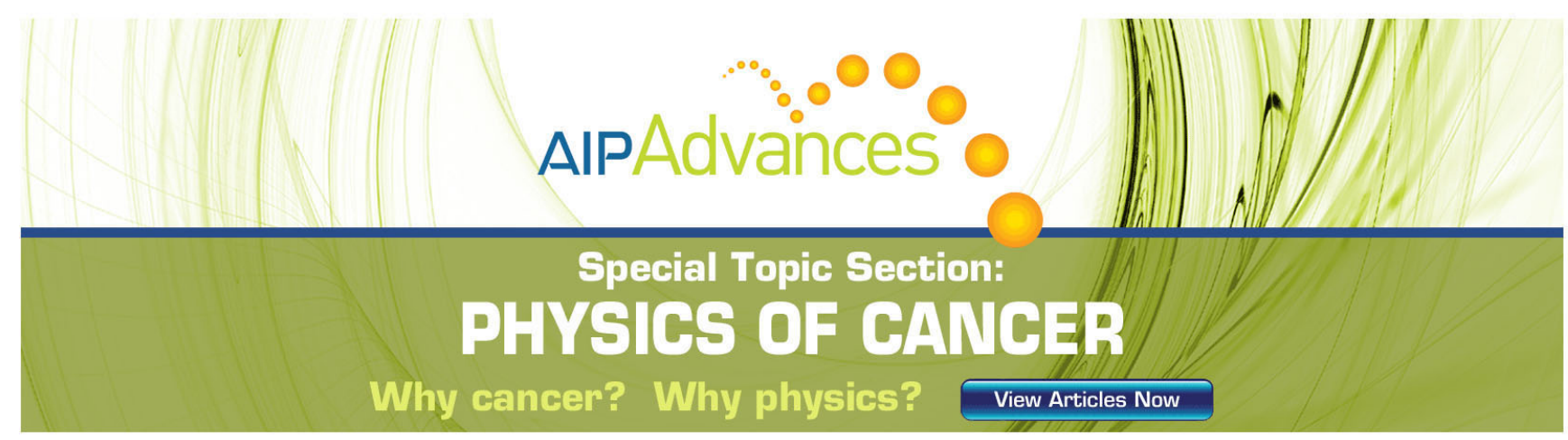




\title{
Origin of thickness dependent dc electrical breakdown in dielectrics
}

\author{
George Chen, ${ }^{1,2}$ Junwei Zhao, ${ }^{1}$ Shengtao $\mathrm{Li}^{2}{ }^{2}$ and Lisheng Zhong ${ }^{2}$ \\ ${ }^{1}$ Faculty of Physical and Applied Sciences, University of Southampton, Southampton, United Kingdom \\ ${ }^{2}$ State Key Laboratory of Electrical Insulation and Power Equipment, Xi' an Jiaotong University, Xi' an, China
}

(Received 3 April 2012; accepted 10 May 2012; published online 31 May 2012)

\begin{abstract}
A model based on space charge dynamics under high dc electric field has been proposed to explain commonly observed thickness dependent breakdown of polymeric material. The formation and dynamics of space charge will result in local electric field enhancement that has a direct impact on dielectric breakdown. The simulation results show that the breakdown depends on the sample thickness with a power index of 0.143 , indicating the space charge and its dynamics are responsible for thickness dependent breakdown. The model also predicts the effect of voltage ramping rate on the electrical breakdown strength. (C) 2012 American Institute of Physics. [http://dx.doi.org/10.1063/1.4721809]
\end{abstract}

High electric field breakdown and ageing failure of insulating materials have been important phenomena in both electronic and electrical industries. Much effort has been spent to understand the breakdown processes as they are not only scientifically challenging but also practically important. Several different mechanisms have been proposed including electronic, avalanche, electromechanical, thermal breakdowns, and partial discharges. ${ }^{1}$ One of the important observations during breakdown studies is that the electrical breakdown strength of solid dielectrics has been found to decrease with the increase in sample thickness. An inverse power law is generally reported ${ }^{2}$

$$
E(d)=k d^{-n},
$$

where $\mathrm{E}$ is the applied electric field at breakdown and $\mathrm{k}$ and $\mathrm{n}$ are two constants that are associated with the testing material.

The relationship described in the above equation is purely empirical and obtained from many electrical breakdown experiments of different dielectrics under ac, dc, and impulse conditions. Various attempts have been made to understand why such a relationship exists. Early work reviewed by Forlani and Minnaja ${ }^{3}$ revealed that for inorganic materials such as $\mathrm{NaCl}$ single crystal, the power index $n$ changes from 0.5 for thinner thickness to 0.25 for thicker thickness. While for polycrystalline materials such as $\mathrm{Al}_{2} \mathrm{O}_{3}$ and $\mathrm{SiO}_{2}, n$ varies in the opposite way with sample thickness. Different mechanisms were proposed to explain the change in power index. It is generally acknowledged that the breakdown event may be controlled by defects presented in the material. The characteristics of breakdown should be described by the Weilbull statistics. The most common explanation is to extend the applicability of the Weibull statistics by including volume effect. ${ }^{4}$ The number of defects in the material increases with the volume. By fixing the testing area, it is reasonable to expect an inverse power relationship between the electrical breakdown strength and sample thickness. Following the same argument, it can also be derived that a similar relationship is true for the electrical breakdown strength and testing area with the same power index when the sample thickness is fixed. However, there are clear exper- imental evidences showing the power index is very different, ${ }^{5,6}$ indicating invalidity of the argument.

Review by Boggs ${ }^{7}$ on the issue of space charge in polymeric dielectrics has shown that the power index in Eq. (1) should be 0.5 following consideration of energy criteria upon breakdown. The role of space charge in electric performance was considered but not as a dominant factor. It has been pointed out that the other factors such as material manufacturing processes may influence the value of $n$. On the other hand, when considering the thickness dependent dielectric breakdown of silicon dioxide that is widely used as insulation in IC devices, Zhou et al. ${ }^{8}$ have realized the importance of charge dynamics in the process of breakdown and proposed a dynamic electron trapping-detrapping process in the material under the application of an electric field. It has been proposed that the conductive path (breakdown) occurs when a critical electron trap density is reached. This can only happen when the trapping rate exceeds the detrapping rate. The model also predicts a threshold thickness below which no breakdown should take place because the electron detrapping rate is greater than the trapping rate. Unfortunately, there is no strong experimental evidence to support the existence of the threshold thickness. Additionally, it is not clear why trapping and detrapping rates change with the sample thickness.

Percolation theory has been proposed to dielectric breakdown. ${ }^{9}$ Recently, Wu et al. ${ }^{10}$ have proposed a percolation model to describe electric breakdown and ageing in polymers as containing a 3D lattice of electron trap sites. A range of barriers dependent of electric fields were set to simulate electron transfer. In the higher electric field, the sites are connected to form clusters due to the reduction in potential barrier. Further increases in electric field may lead to percolation of the clusters causing breakdown. Recently, they have used the field-assisted proposed percolation model to simulate statistical behavior of electrical breakdown in insulating polymers and the effect of area on breakdown strength has been studied. The thickness effect has not been addressed.

It is clear from the literature review that thickness dependent dielectric breakdown is a common phenomenon but the detailed mechanisms are poorly understood. All the 
proposed models lack creditable experimental support and are therefore difficult to relate to physical processes taking place in the material under higher electric fields. In the consideration of reliable operation of electronic devices and high voltage apparatus, it is extremely important to understand the underlying physical processes under high electric fields.

The electrical breakdown in solid dielectric materials is directly related to charge injection and space charge dynamics. It has been shown ${ }^{11}$ when a steady dc voltage is applied to the fixed sample, thickness charge injection takes place and positive charge packet forms and it moves towards the cathode but stops at a certain position when the applied dc field is higher. The final positive charge packet position towards the cathode increases with the applied field. Recently, it has been experimentally demonstrated ${ }^{12}$ that the positive charge mobility decreases with the applied electric field in the high field region. Considering the characteristics of positive charge mobility, we have modeled the positive charge packet formation and dynamics in polymeric material based on the bipolar charge injection model. Additionally, the experimental results from polyethylene under constant $\mathrm{dc}$ voltages have shown the charge injection and charge packet. ${ }^{11}$ It is interesting to notice that the breakdown occurs when the local electric field reaches a constant value of $550 \mathrm{kV} / \mathrm{mm}$. This strongly suggests that the material has an "intrinsic breakdown strength" at which the breakdown occurs. It also suggests that space charge plays an important role in breakdown event as its presence determines the local electric field.

Based on these strong experimental evidences, we propose the following electric breakdown model. The breakdown under dc condition is governed by space charge injected from the electrodes and injected charges are responsible for electric field enhancement in the bulk. Once the local field reaches the intrinsic breakdown strength of the material, the electrical breakdown occurs. The bipolar charge injection model widely used for space charge simulation and high field phenomena ${ }^{13}$ is equally applicable here.

Charge transportation in dielectrics is essentially governed by a set of basic equations. They describe the behaviour of charge carriers in the system through a time and space dependent total flux $j(x, t)$ by neglecting diffusion:

Transport equation

$$
j_{C}(x, t)=\mu n(x, t) E(x, t) .
$$

Continuity equation

$$
\frac{\partial n(x, t)}{\partial t}+\frac{\partial j(x, t)}{\partial x}=s .
$$

Poisson's equation

$$
\frac{\partial E(x, t)}{\partial x}=\frac{\rho(x, t)}{\varepsilon},
$$

where $\mu$ is the mobility of carriers, $\mathrm{n}$ the density of mobile species, E the electric field, $\mathrm{j}$ the current density, $\mathrm{x}$ the spatial coordinate, $\mathrm{t}$ the time, $\mathrm{s}$ the source term, $\varepsilon$ the dielectric permittivity, and $\rho$ the net charge density.
In this bipolar charge model charge carriers are injected from the electrodes, electrons from the cathode, and holes from the anode. Injection occurs based on the Schottky mechanism whereby overcoming a potential barrier at the interfaces. After penetrating into the material, the carriers, under the influence of the applied field, will drift across the material characterized by an effective mobility. Throughout its motions, some carriers are trapped in the localized states, i.e., deep trap centres and therefore, the total amount of charges moving across reduces. However, no extraction barrier is introduced in the model. On the other hand, positive and negative charges are prone to recombine with their opposite species (electrons with holes).

In our initial attempt, the parameters from our previous simulation of bipolar charge mode ${ }^{14}$ were adopted. The mobility for holes at a higher electric field has been extended using curve fitting. The material used in the simulation is low density polyethylene with a thickness range from 25 to $250 \mu \mathrm{m}$. Based on the existing experimental results from the literature, ${ }^{11}$ the intrinsic breakdown strength for polyethylene is set to be $550 \mathrm{kV} / \mathrm{mm}$, i.e., when the internal local field in the material reaches $550 \mathrm{kV} / \mathrm{mm}$, the simulation stops. Then the applied electric field is obtained based on the applied voltage for a particular sample thickness.

Figure 1 shows simulation results for different sample thicknesses when the dc voltage rise rate is $300 \mathrm{~V} / \mathrm{s}$. The same bipolar charge model widely employed to simulate dynamics of charge packet ${ }^{15}$ was used in the present simulation with a field dependent velocity showing in Figure 2 being used. The velocity curve has been extended to much higher field region based on the results from literature. ${ }^{9}$ The negative differential mobility (Gunn effect) is a well known phenomenon in semiconductors. However, for polymeric material such as polyethylene the microstructure and its associated energy diagram are very different. Lewis et al. ${ }^{16}$ have proposed a different mechanism based on a tunneling concept of holes between molecular chains for negative differential mobility. The negative differential mobility in polyethylene has been observed by us. ${ }^{12}$ The values for other parameters in the model have been kept the same as that in the previous simulation.

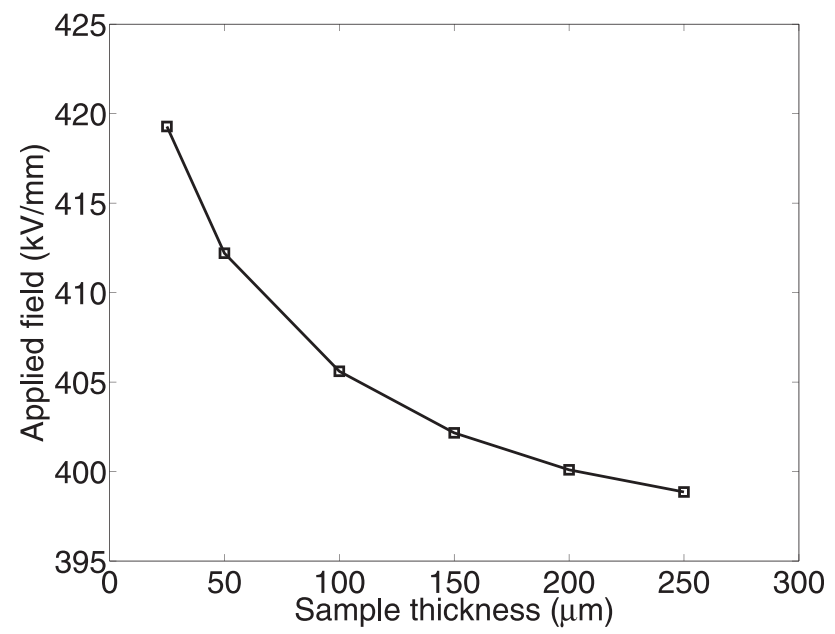

FIG. 1. Breakdown strength versus sample thickness in polyethylene, clearly indicating the measured breakdown strength decreases with sample thickness. 


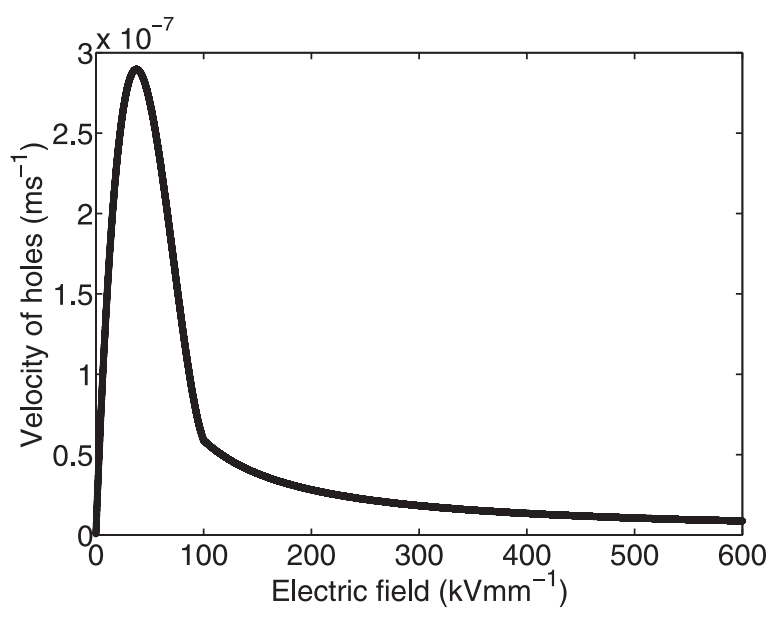

FIG. 2. Positive charge velocity versus the electric field used in the simulation. The first part of velocity up to $100 \mathrm{kV} / \mathrm{mm}$ is based on our own measurements $^{12}$ and the velocity in high field region was obtained from Ref. 11.

It is obvious that the breakdown strength decreases with sample thickness. The decreasing rate of electric breakdown field reduces with the sample thickness. Considering generally observed inverse power law the data in Figure 1 has been re-plotted with a logarithmic scale as shown in Figure 3. It becomes clear that a liner relationship between the electric breakdown field (applied) and the sample thickness can be obtained with the power index $n=0.143$.

It has been noticed that the linear relationship holds for the other rise rates but the breakdown field changes. Figure 4 shows the effect of a voltage rise rate from $100 \mathrm{~V} / \mathrm{s}$ to $400 \mathrm{~V} /$ $\mathrm{s}$ on the electric breakdown field for $100 \mu \mathrm{m}$ thick polyethylene sample. It is evident that the breakdown field increases with the voltage rise rate. A similar trend for the electric breakdown strength has been observed experimentally, further validating the present model.

The proposed model also allows one to view charge dynamics and electric field evolution prior to the breakdown and electric field distribution. Figures 5 and 6 show charge distribution and electric field distribution at various times

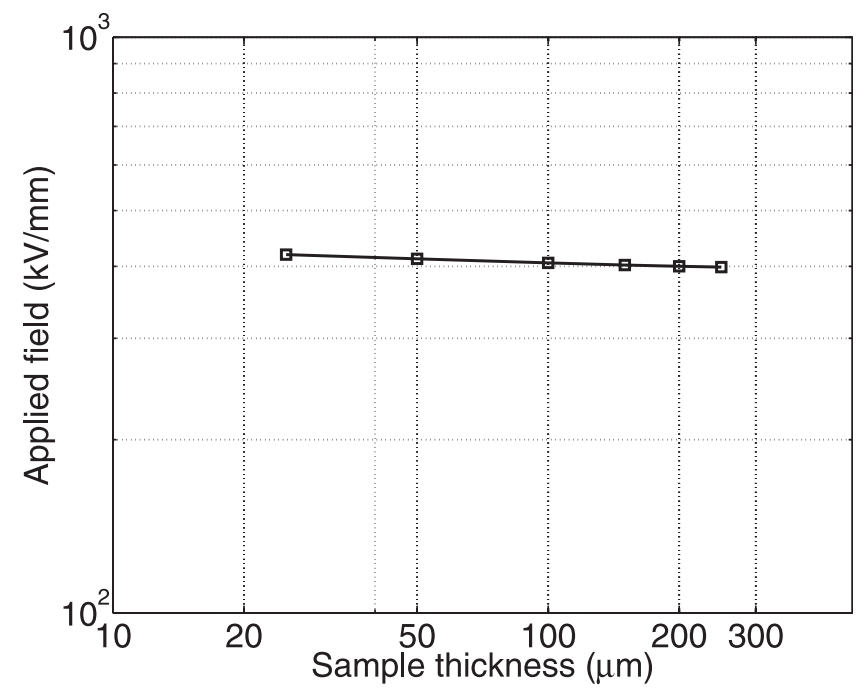

FIG. 3. Logarithmic representation of the simulated data in Figure 1 showing a linear relationship. The power index $n=0.143$ is obtained from the slope of the line.

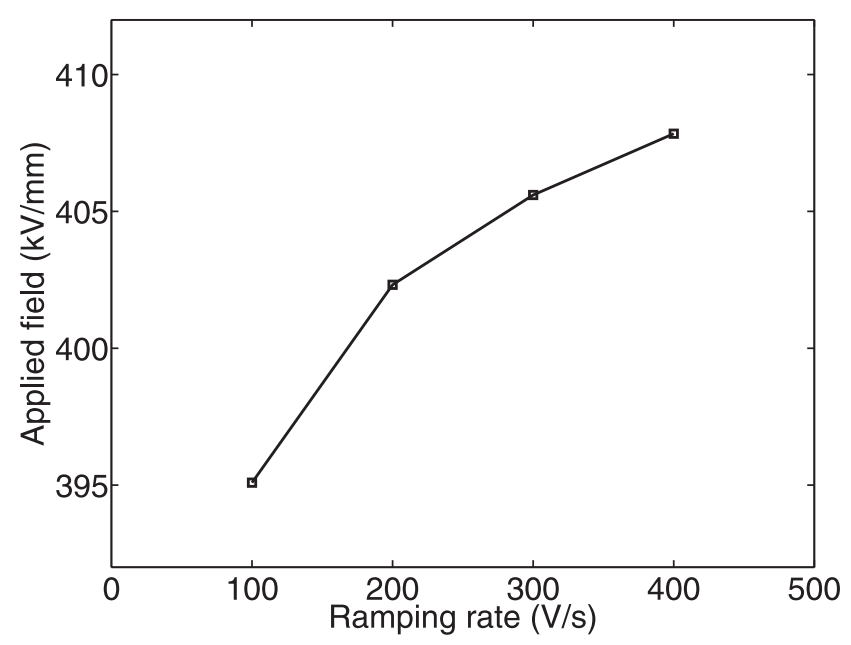

FIG. 4. Influence of voltage rise rate on the electric breakdown strength, indicating the voltage ramping rate affects the measured breakdown strength.

during the voltage raise stage with the last one showing the local electric field reaches a set value of $550 \mathrm{kV} / \mathrm{mm}$. The simulation results are obtained from $100 \mu \mathrm{m}$ thick sample and the voltage rise rate is $300 \mathrm{~V} / \mathrm{s}$.

From Figure 5, it can be seen that a positive charge packet has been developed during voltage rise stage and its magnitude increases with time and its position moves towards the cathode with time. Negative charges are more spread due to a high charge velocity used in the model. It has been noticed that the charge dynamics are different from that under steady dc voltage where the charge packet is further into the bulk. This is related to the fact that a rise voltage is simulated to mimic the dc breakdown test. It has been noticed that when the voltage rise rate is low the positive charge packet moves deep into the bulk at the time when the breakdown occurs.

As the electric field inside the sample is determined by the applied electric field and the contribution from space charge, therefore, the electric field distribution shown in Figure 6 is expected. At the beginning, the applied voltage is very low and there is no charge injection. Consequently, the electric field is purely determined by the applied voltage,

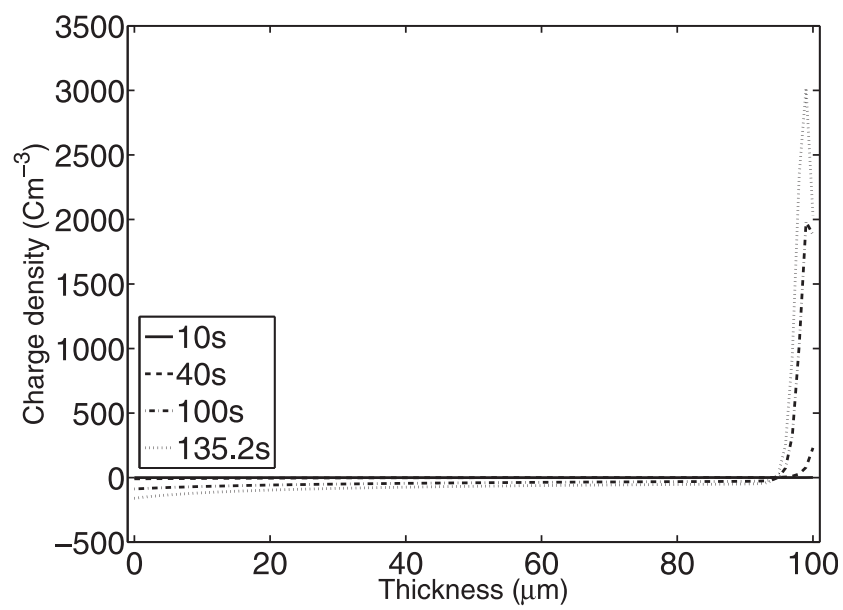

FIG. 5. Space charge dynamics across the bulk of the sample showing positive charge front moves slowly into the bulk of the sample during voltage rise stage. 


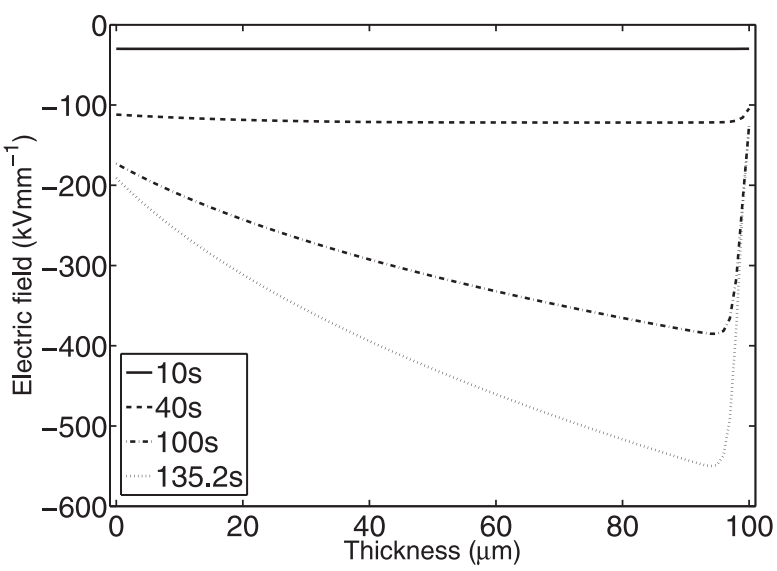

FIG. 6. Electric field distribution across the sample during voltage rise stage indicating the maximum electric field moves slowly into the bulk of the sample.

resulting in a uniform distribution. However, at a later time the applied electric field is high enough and charge injection takes place; the contribution from space charge becomes increasingly important. The maximum electric field increases with the applied voltage (time) and moves from the anode towards the cathode. The maximum electric field always occurs in the bulk of the sample; this suggests that the breakdown under dc condition is initiated from the bulk.

The thickness dependent breakdown of material under dc condition has been simulated based on the space charge model. Several important aspects observed during experimental breakdown tests have been simulated, indicating the above model is appropriate. The range of breakdown obtained from the simulation is very reasonable compared with the experimental data.

The thickness dependent electric breakdown strength occurs for ac, dc, and impulse voltages. The power index $n$ obtained from our simulation is on the lower side of reported value. This is to say that the simulated thickness dependent electric breakdown strength is not as strong as those observed experimentally. Recent report ${ }^{17}$ on impulse breakdown of poly(methyl methacrylate) (PMMA) shows an even lower power index $(\mathbf{n}=\mathbf{0 . 1 2 5})$ which is similar to the value for several other polymers such as polypropylene (PP), polyethylene (PE), and polytetrafluoroethylene (PTFE). Currently, there is limited dc breakdown data for polymeric materials while ac breakdown dependence is much more abundant. There may be different reasons but of one of them could be due to the selection of parameters in the model. Although the values for majority parameters are consistent with those appeared in many published work, it is very difficult to justify their selections such as electron mobility, trapping coefficient and recombination coefficient etc. On the other hand if the material is more homogeneous, i.e., less defects, a lower power index is expected.

The model does not consider the effect of surface. It has been mentioned that the surface will play important role when the thickness of sample gets thinner. ${ }^{3}$ In this case, the effect of space charge on breakdown strength of the thinner material can be overtaken by surface defects, leading to a different relationship with sample thickness.

Breakdown scattering observed in experiment is not mimicked in the simulation. This is because the material in the simulation is considered as homogeneous, therefore, charge formation and dynamics are governed by a unique set of equations, leading to a unique result. In practice there may be various defects in the material, which will affect the charge movement and dynamics, hence causing scattering in the final breakdown result. It has been experimentally demonstrated in 2D space charge measurement ${ }^{18}$ that space charge is different at the cross section. The inhomogeneity is, therefore, the cause for breakdown scattering in a material.

In summary, a model for thickness dependent electric breakdown under dc conditions has been proposed. The model is based on strong experimental evidence of bipolar charge injection and the formation of charge packet under higher electric fields. From the simulation it is clear that the thickness dependent dielectric breakdown is the result of charge dynamics in the material. In addition, the model also shows correctly the relationship between the dielectric breakdown strength and dc voltage rise rate.

This is our initial attempt and the simulation based on generally accepted parameters of the bipolar charge model has shown a promising result. We are working on the charge measurements during voltage rise stage and the results will provide us more evidence about charge dynamics during voltage rise stage up to pre-breakdown.

This research has been supported by the State Key Laboratory of Electrical Insulation and Power Equipment, Xi'an Jiaotong University, China (EIEP12211).

${ }^{1}$ V. A. Zakrevskii, N. T. Sudar, A. Zaopo, and Yu. A. Dubitsky, J. Appl. Phys. 93, 2135 (2003).

${ }^{2}$ H. K. Kim and F. G. Shi, IEEE Trans. Dielectr. Electr. Insul. 8(2), 248 (2001).

${ }^{3}$ S. Boggs, IEEE Electr. Insul. Mag. 20(4), 22 (2004).

${ }^{4}$ J. Artbauer, Electrotech Z 91, 326 (1970).

${ }^{5}$ S. Cygan and J. B. Laghari, IEEE Trans. Electr. Insul. 22(6), 835 (1987).

${ }^{6}$ S. J. Laihonen, U. Gafvert, T. Schutte, and U. W. Gedde, IEEE Trans. Dielectr. Electr. Insul. 14, 275 (2007).

${ }^{7}$ F. Forlani and N. Minnaja, J. Vac. Sci. Technol. 6(4), 518 (1969).

${ }^{8}$ H. Zhou, F. G. Shi, B. Zhao, Microelectron. J. 34, 259 (2003).

${ }^{9}$ P. D. Beale and P. M. Duxbury, Phys. Rev. B 37, 2785 (1988).

${ }^{10}$ K. Wu, Y. Wang, Y. H. Cheng, L. A. Dissado, and X. J. Liu, J. Appl. Phys. 107, 064107 (2010).

${ }^{11}$ K. Matsui, Y. Tanaka, T. Takada, T. Fukao, K. Fukunaga, T. Maeno, and J. M. Alison, IEEE Trans. Dielectr. Electr. Insul. 12, 406 (2005).

${ }^{12}$ G. Chen and J. Zhao, J. Phys. D: Appl. Phys. 44(21), 212001 (2011).

${ }^{13} \mathrm{G}$. Chen, in ISEIM (The Institute of Electrical Engineers of Japan, Kyoto, Japan, 2011), p. 156.

${ }^{14}$ J. Zhao, Z. Xu, G. Chen, and P. L. Lewin, J. Appl. Phys. 108(12), 124107 (2010).

${ }^{15}$ J. Zhao, G. Chen, and P. L. Lewin, "Investigation into the formation of charge packets in polyethylene: Experiment and Simulation," J. App. Phys. (submitted).

${ }^{16}$ J. P. Jones, J. P. Llewellyn, and T. J. Lewis, IEEE Trans. Dielectr. Electr. Insul. 12, 951 (2005).

${ }^{17}$ L. Zhao, G. Z. Liu, J. C. Su, Y. F. Pan, and X. B. Zhang, IEEE Trans. Plasma Sci. 39(7), 1613 (2011).

${ }^{18}$ M. Fukuma, K. Fukunaga, and C. Laurent, Appl. Phys. Lett. 88, 253110 (2006). 